\title{
ON THE TWENTY-NINTH QUESTION OF ALLEN SHIELDS
}

\author{
JOSEPH G. STAMPFLI ${ }^{1}$
}

\begin{abstract}
Not every hyponormal weighted shift is the sum of a subnormal weighted shift (in the same basis) and a trace class operator.
\end{abstract}

In [1, p. 117] Allen Shields raises the following question (\#29): Is every hyponormal weighted shift the sum of a subnormal weighted shift and a trace class operator? (Both shifts are defined with respect to the same basis.) We will show that the answer is no. Indeed there exists a hyponormal weighted shift which is not the sum of a subnormal weighted shift and an operator of Schatten $p$-class for any $p$ where $1 \leqslant p<2$. We would conjecture that the restriction on $p$ in the previous statement is unnecessary.

We will adhere to the notation from [1]. Thus, if $\left\{e_{n}\right\}$ is an orthonormal basis for the Hilbert space $H$, then the operator $T$ defined by $T e_{n}=a_{n} e_{n+1}$ for $n=1,2, \ldots$ is a weighted shift with weights $\left\{a_{n}\right\}$. For such an operator $T$ the $p$-norm is

$$
\|T\|_{p}=\left[\sum_{n=1}^{\infty}\left|a_{n}\right|^{p}\right]^{1 / p} .
$$

LEMMA. Let $S$ be a subnormal weighted shift with weights $\left\{a_{n}\right\}$ where the $a_{n}$ 's are positive, $c_{n}=a_{n}^{2}$ and $\lim _{n \rightarrow \infty} a_{n}=1$. Then

$$
c_{j+2}-c_{j+1} \leqslant \frac{c_{n}}{c_{j}}\left[1+\frac{c_{n+1}-c_{n}}{c_{n}-c_{j}}\right]\left(c_{j+1}-c_{j}\right)
$$

for $j \leqslant n$. If

(1) $d / 2<\left(c_{n}-c_{k}\right)<d<\frac{1}{2}$ for $j \leqslant k \leqslant N$,

(2) $\left(c_{n+1}-c_{n}\right)<d^{2} / 4$, and

(3) $c_{j} \geqslant 4 / 5$,

then

$$
a_{N}-a_{j} \leqslant\left[\left(a_{j+1}-a_{j}\right) / 2 d\right] e^{2(N-j) d} .
$$

Proof. The first relation is derived from the necessary condition I of Theorem 4 of [2]. As in the proof of Theorem 5 in that paper, we are led to the following variant of equation (6) of [2]:

Received by the editors September 14, 1976.

AMS (MOS) subject classifications (1970). Primary 47B20.

Key words and phrases. Subnormal, hyponormal, weighted shift, Schatten $p$-class.

'Supported in part by a grant from the National Science Foundation MCS \# 76-03594.

○ American Mathematical Society 1977 


$$
c_{n+1} \geqslant c_{j+1}+c_{j} c_{j+1} \frac{c_{j+2}-c_{j+1}}{c_{j+1}-c_{j}}\left[\frac{1}{c_{j}}-\frac{1}{c_{n}}\right]
$$

which is valid for $j \leqslant n$. The (weaker) first inequality now follows easily from this. To obtain the second inequality, we substitute in the first one to obtain

$$
c_{j+2}-c_{j+1} \leqslant(1+2 d)\left(c_{j+1}-c_{j}\right) \text {. }
$$

Thus

$$
\begin{aligned}
c_{N}-c_{j} & =\sum_{k=j}^{N-1}\left(c_{k+1}-c_{k}\right) \leqslant\left(c_{j+1}-c_{j}\right) \sum_{k=0}^{N-j}(1+2 d)^{k} \\
& \leqslant \frac{\left(c_{j+1}-c_{j}\right)}{2 d}(1+2 d)^{N-j} \leqslant \frac{\left(c_{j+1}-c_{j}\right)}{2 d} e^{2(N-j) d} .
\end{aligned}
$$

To complete the proof we need only observe that $c_{N}-c_{j}=a_{N}^{2}-a_{j}^{2}$ and similarly for $c_{j+1}-c_{j}$.

The EXAMPLE. First we give a rough description of our hyponormal weighted shift. It will have a long string of constant weights, then jump up to a new level where again there will be a long string of constant weights and so on. We will refer to the value of these long strings of constant weights as levels. Thus the value of all weights at the $k$ th level will be $l_{k}$.

Let

$$
\gamma=\sum_{n=2}^{\infty} n^{-1}(\log n)^{-2}
$$

Set

$$
l_{k}=\gamma^{-1} \sum_{n=2}^{k-1} n^{-1}(\log n)^{-2} \quad \text { for } k \geqslant 3
$$

and set

$$
d_{k}=l_{k+1}-l_{k}=\gamma^{-1} k^{-1}(\log k)^{-2} \text {. }
$$

We set $l_{1}=l_{2}=0$ and $d_{1}=d_{2}=1$. At the $k$ th level our shift will have all weights equal to $l_{k}$, and the length of the string or number of weights at the $k$ th level will be $3 d_{k}^{-1}$ (the fact that this last number is not an integer should disturb no one). The weighted shift we have just defined is clearly hyponormal of norm 1 . Let us call this operator $T$, and say its weights are $\left\{b_{n}\right\}$. Thus $\lim _{n \rightarrow \infty} b_{n}=1$. We wish to show that $T$ is not the sum of any subnormal weighted shift and an operator of Schatten $p$-class for $1 \leqslant p<2$.

The strategy of attack is the following. Let $S$ be a subnormal weighted shift with weights $\left\{a_{n}\right\}$. If the weights $\left\{a_{n}\right\}$ fit closely to the $\left\{b_{n}\right\}$ at the $k$ th level $(k$ odd) then they must miss badly at the $(k+1)$ st level. In other words the string of constant weights has been chosen long enough so that our putative subnormal weighted shift must have two adjacent weights very close together at the middle of a level, but short enough so that, given these two adjacent weights, by our Lemma the weights cannot have climbed sufficiently close to 
the next level by the time we reach that next string to allow a $c_{p}$ perturbation. A crucial link in the proof depends on the convergence of the series $\Sigma d_{k}$ versus the divergence of $\sum d_{k}^{p-1}$ for $1 \leqslant p<2$.

To implement our strategy we now look carefully at the $k$ th level which we divide into 3 equal parts each of length $d_{k}^{-1}$ as follows.

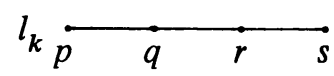

We assume $k$ is so large that $l_{k} \geqslant 5 / 6$.

ClaIm. We may assume the weights of $S$ satisfy the inequality $l_{k}-d_{k} 10^{-8}$ $\leqslant a_{n} \leqslant l_{k}+d_{k} 10^{-8}$ for $q \leqslant n \leqslant r$. We use the constant $10^{-8}$ at all levels. For if not, say $a_{r} \geqslant l_{k}+10^{-8} d_{k}$. Then $a_{n}-b_{n} \geqslant d_{k} 10^{-8}$ for $r \leqslant n \leqslant s$ and, since $s-r=d_{k}$, the contribution to the $p$-norm of $T-S$ from the $k$ th level alone is $10^{-8 p} d k^{-1}$. Since $\sum d_{k}^{-1}=\infty$ for $1 \leqslant p<2$ we would be done. Thus we may assume that $\left|l_{k}-a_{n}\right| \leqslant 10^{-8} d_{k}$ for $q \leqslant n \leqslant r$. Since in this case $a_{r}-a_{q} \leqslant 2 \cdot 10^{-8} d_{k}$, there must exist a $j$ between $q$ and $r$ such that $\left(a_{j+1}-a_{j}\right) \leqslant 2 \cdot 10^{-8} d_{k}^{2}$. Applying the same argument to the weights at the $(k+1)$ st level we see that there must exist a weight $a_{n}$ in the middle third of that level such that

$$
a_{n+1}-a_{n} \leqslant 2 \cdot 10^{-8} d_{k+1}^{2} .
$$

We now consider $n, j$ and $k$ fixed and apply the Lemma. Note that

$$
c_{n+1}-c_{n} \leqslant 2\left(a_{n+1}-a_{n}\right) \leqslant 4 \cdot 10^{-8} d_{k+1}^{2}
$$

and

$$
c_{j+1}-c_{j} \leqslant 2\left(a_{j+1}-a_{j}\right) \leqslant 4 \cdot 10^{-8} d_{k}^{2}
$$

Moreover,

$$
c_{n}-c_{j} \leqslant 2\left(a_{n}-a_{j}\right) \leqslant 3 d_{k} .
$$

Thus if we set $d=3 d_{k}$ then

$$
c_{n+1}-c_{n} \leqslant 4 \cdot 10^{-8} d_{k+1}^{2} \leqslant d_{k}^{2} \leqslant d^{2} / 4 .
$$

We now apply the Lemma with $d=3 d_{k}$ to obtain

$$
a_{N}-a_{j} \leqslant \frac{2 d_{k}^{2}}{10^{8}} \cdot \frac{1}{6 d_{k}} e^{6(N-j) d_{k}} .
$$

If we now take $N-j=3 d_{k}^{-1}$ then

$$
a_{N}-a_{j} \leqslant \frac{d_{k}}{3 \cdot 10^{8}} e^{18} \leqslant \frac{d_{k}}{3} .
$$

But the last inequality implies that $b_{m}-a_{m} \geqslant d_{k} / 2$ for every weight $a_{m}$ in the first part of the string at the $(k+1)$ st level, where this first part contains at least $d_{k}^{-1}$ terms. Thus in this case, the contribution to the $p$-norm of $T-S$ from the terms at the $(k+1)$ st level must exceed $2^{-p} d_{k}^{p-1}$.

To summarize, in the two cases considered we have shown that the $k$ and 
$(k+1)$ st levels considered jointly must contribute a term to the $p$-norm of $T-S$ of magnitude constant $\cdot d_{k}^{p-1}$. Since $\sum d_{k}^{p-1}=\infty$ for $1<p<2$ we are finished.

\section{REFERENCES}

1. A. L. Shields, Weighted shift operators and analytic function theory, Topics in Operator Theory, Math. Surveys, no. 13, Amer. Math. Soc., Providence, R. I., 1974.

2. J. G. Stampfli, Which weighted shifts are subnormal?, Pacific J. Math. 17 (1966), 356-379.

Department of Mathematics, Indiana University, Bloomington, Indiana 47401 